\title{
SQUARE COMPACTNESS AND THE FILTER EXTENSION PROPERTY
}

\author{
DAVID BUHAGIAR AND MIRNA DŽAMONJA
}

\begin{abstract}
We show that the consistency strength of $\kappa$ being $2^{\kappa}$-square compact is at least weak compact and strictly less than indescribable. This is the first known improvement to the upper bound of strong compactness obtained in 1973 by Hajnal and Juhász.
\end{abstract}

\section{INTRODUCTION}

In their 1973 paper [2], András Hajnal and István Juhász introduced a large cardinal property based on the productivity of $\kappa$-compactness, the square compact cardinals. Following and expanding on [2] we define that notion:

Definition 1.1. An infinite cardinal $\kappa$ is said to be $\lambda$-square compact if for any $(<\kappa)$-compact space $X^{1}$, with $w(X) \leq \lambda$, the square $X^{2}=X \times X$ is also $(<\kappa)$-compact.

$\kappa$ is said to be square compact if it is $\lambda$-square compact for all $\lambda$ and weak square compact if it is $\kappa$-square compact.

It is not difficult to see that if $\kappa$ is $\lambda$-square compact, and $X, Y$ are $(<\kappa)$ compact spaces of weight $\leq \lambda$, then $X \times Y$ is also $(<\kappa)$-compact. Indeed, first note that in these circumstances the space $X \oplus Y$ is $(<\kappa)$-compact with weight $\leq \lambda$. Consequently, $(X \oplus Y) \times(X \oplus Y)$ is also $(<\kappa)$-compact whenever $\kappa$ is $\lambda$-square compact. But $X \times Y$ is a closed subspace of $(X \oplus Y) \times(X \oplus Y)$, and therefore is $(<\kappa)$-compact.

In [2] the authors prove that a square compact cardinal is weakly compact. It is easy to see and was certainly known to the authors of [2] that a $\lambda$-strongly compact cardinal is $\lambda$-square compact. The exact consistency strength of the notion of $\lambda$-square compactness and square compactness, in particular if the latter is equivalent to weak compactness has been an open problem since [2]. In this paper we obtain a much better upper bound for the consistency strength of $\lambda$-square compact cardinals $\kappa$ for $\lambda \leq 2^{\kappa}$, showing

2010 Mathematics Subject Classification. Primary 03E55, 54D20; Secondary 54F05.

Key words and phrases. square compact cardinal, box topology, filter extension property.

${ }^{1}$ We note that for simplicity we only work with Hausdorff spaces. 
that it is consistent modulo large cardinals that the first weakly compact cardinal $\kappa$ is $2^{\kappa}$-square compact (Theorem 6.1(2)). In particular, for such $\lambda$ there cannot be any ZFC implication from being $\lambda$-square compact to being indescribable, measurable (or any other large cardinal property that cannot be exhibited by the first weakly compact cardinal), yet alone to being $\lambda$-strongly compact. We also show that inaccessible $\kappa$-square compact cardinals are already weakly compact (Theorem 6.1(1)).

Surprisingly enough, our method does not apply at all to fully square compact cardinals $\kappa$ or to those that are $\lambda$-square compact for $\lambda>2^{\kappa}$ : the best known upper bound for the consistency strength of them after this work still remains a strongly compact cardinal.

The most interesting case for our work is when $\lambda^{<\kappa}=\lambda$. We go through introducing the notion of $\lambda$-filter extension property for $\kappa$ and showing that it implies $\kappa$ being $\lambda$-square compact (Theorem 3.6) and is equivalent to $\kappa$ being nearly $\lambda$-strongly compact (Theorem 4.2), which is a large cardinal notion introduced by White in [12]. This allows to make a connection with Schanker's notion of nearly $\lambda$-supercompactness, whose consistency strength was studied in [1] and whose results we use to obtain our upper bound.

A side gain of our work are numerous equivalences obtained for the notion of $\lambda$-filter extension property, including topological ones (Theorem 3.2 ) and the elementary embedding ones (Theorem 3.6), which then lead to various equivalences of weak compactness (Theorem 5.1). The topological equivalences use the $(<\kappa)$-box topology and lead to a better understanding of it. For example, $\kappa$ has the $\lambda$-filter extension property if and only if $2^{\lambda}$ is $(<\kappa)$-compact in the $(<\kappa)$-box topology (Theorem 3.2).

\section{Notation, CONVEntions And OBSERVATions}

Throughout our work we shall keep the convention that $2=\{0,1\}$ and that for any set $X$, any subset of $X$ is identified with its characteristic function. Furthermore the set of all functions from $X$ to 2 identified with the product $2^{X}$, so we have a triple identification between $\mathcal{P}(X),{ }^{X} 2$ and $2^{X}$.

Greek letters $\kappa$ and $\lambda$ will always stand for infinite cardinal numbers.

Recall that a filter $\mathcal{F}$ on a set $X$ is said to be principal if it is of the form $\{A \subseteq X: Y \subseteq A\}$ for some $\emptyset \neq Y \subseteq X$, in which case $Y=\bigcap \mathcal{F}$. Otherwise, it is non-principal (and hence $\bigcap \mathcal{F}=\emptyset$ ). A filter is said to be a proper filter if $\mathcal{F} \neq \mathcal{P}(X)$. For a regular cardinal $\kappa$, we say that a filter $\mathcal{F}$ is $(<\kappa)$-complete if $\bigcap \mathcal{A} \in \mathcal{F}$ for every family $\mathcal{A} \subseteq \mathcal{F}$ of size less than $\kappa$. It 
is not difficult to see that if $\mathcal{F}$ is a non-principal $(<\kappa)$-complete filter on some set $X$, then every set in $\mathcal{F}$ has size at least $\kappa$.

Let $X$ be an infinite set and $\mathcal{F}_{0}$ a non-principal $(<\kappa)$-complete filter on $X$. In some of our proofs the objective will be to obtain a $(<\kappa)$-complete filter $\mathcal{F}$ on $X$ extending $\mathcal{F}_{0}$ and having some special property. We give the following definition with this purpose in mind.

Definition 2.1. Let $\mathcal{F}$ be a filter on a set $X$. Consider the set $G(\mathcal{F})$, the grill associated with $\mathcal{F}$, of all subsets $S$ of $X$ such that $S$ meets every $F \in \mathcal{F}$. One observes that

$$
S \in G(\mathcal{F}) \backslash \mathcal{F} \quad \text { if and only if } \quad X \backslash S \in G(\mathcal{F}) \backslash \mathcal{F} .
$$

Therefore, $\mathcal{F}=G(\mathcal{F})$ if and only if $\mathcal{F}$ is an ultrafilter and

$$
G(\mathcal{F})=\{S \subseteq X: X \backslash S \notin \mathcal{F}\} .
$$

Note that $\mathcal{F} \subseteq G(\mathcal{F})$ and that $G(\mathcal{F})$ satisfies the following conditions:

(1) $\emptyset \notin G(\mathcal{F})$.

(2) If $S \in G(\mathcal{F})$ and $S \subseteq T \subseteq X$, then $T \in G(\mathcal{F})$.

(3) If $\mathcal{F}$ is $(<\kappa)$-complete, then for every collection $\left\{S_{\lambda}: \lambda \in \Lambda\right\}$ of subsets of $X$, with $|\Lambda|<\kappa$, if $\bigcup_{\lambda \in \Lambda} S_{\lambda} \in G(\mathcal{F})$, at least one $S_{\lambda}$ is in $G(\mathcal{F})$.

The following is easily verified.

Proposition 2.2. Let $\mathcal{F}_{0}$ be a proper filter on $X$ and $S \subseteq X$ such that neither $S$ nor $X \backslash S$ is in $\mathcal{F}_{0}$. Then

$$
\left\{F \cap L: F \in \mathcal{F}_{0} \text { and } S \subseteq L \subseteq X\right\}
$$

generates a proper filter $\mathcal{F}$ that contains $\{S\} \cup \mathcal{F}_{0}$. Moreover, if $\mathcal{F}_{0}$ is $(<\kappa)$ complete, then so is $\mathcal{F}$.

Definition 2.3. Let $X$ be a set. A subset $\mathcal{A}$ of $\mathcal{P}(X)$ measures a subset $A$ of $X$ if $A \in \mathcal{A}$ or $X \backslash A \in \mathcal{A}$. $\mathcal{A}$ measures a subset $\mathcal{C}$ of $\mathcal{P}(X)$ if $\mathcal{A}$ measures every element $C$ of $\mathcal{C}$.

$\kappa$ is said to have the filter property if for every subset $\mathcal{C}$ of $\mathcal{P}(\kappa)$ of size $\leq \kappa$, there is a $(<\kappa)$-complete non-principal filter $\mathcal{F}$ on $\kappa$ which measures $\mathcal{C}$.

So $\mathcal{F}$ is an ultrafilter on $\kappa$ if it measures $\mathcal{P}(\kappa)$. It is well-known that an uncountable cardinal $\kappa$ has the filter property if and only if $\kappa$ is weakly compact.

For cardinals $\kappa \leq \lambda$ we write $\mathcal{P}_{\kappa}(\lambda)$ for the family $\{a \subseteq \lambda:|a|<\kappa\}$. 
Definition 2.4. A filter $\mathcal{F}$ on $\mathcal{P}_{\kappa}(\lambda)$ is said to be fine if for every $\alpha<\lambda$ we have $B_{\alpha}=\left\{a \in \mathcal{P}_{\kappa}(\lambda): \alpha \in a\right\} \in \mathcal{F}$.

Note that if $\mathcal{F}$ is a fine $(<\kappa)$-complete filter on $\mathcal{P}_{\kappa}(\lambda)$, then $C_{a}=\{b \in$ $\left.\mathcal{P}_{\kappa}(\lambda): a \subseteq b\right\} \in \mathcal{F}$ for every $a \in \mathcal{P}_{\kappa}(\lambda)$. Indeed, $C_{a}=\bigcap_{\alpha \in a} B_{\alpha}$ and $|a|<\kappa$.

We now mention some notions from topology.

It is well known that even for $T_{0}$ spaces $|X| \leq 2^{w(X)}$, which is a fact that we shall use without further mention.

A topological notion connected to the $(<\kappa)$-completeness of filters is that of $(<\kappa)$-compactness.

Definition 2.5. A topological space $X$ is said to be $(<\kappa)$-compact, if every open cover of $X$ has a subcover of cardinality strictly less than $\kappa$.

This notion is sometimes easier to express in terms of the complementary property of closed sets.

Definition 2.6. Let $X$ be a set. A subset $\mathcal{A}$ of $\mathcal{P}(X)$ is said to satisfy the $(<\kappa)$-intersection property if $\bigcap_{i<\gamma} A_{i} \neq \emptyset$ whenever $\gamma<\kappa$ and $\left\{A_{i}: i<\right.$ $\gamma\} \subseteq \mathcal{A}$.

The following observation then connects the notions of compactness and completeness.

Observation 2.7. Suppose that $\kappa$ is a regular cardinal. A topological space $X$ is $(<\kappa)$-compact if and only if every $(<\kappa)$-complete filter consisting of closed sets in $X$ has a nonempty intersection if and only if every family of closed sets with the $(<\kappa)$-intersection property, has a nonempty intersection.

Filters are not only connected to compactness but also to the productiveness of that notion. Let us recall some product topology definitions.

Definition 2.8. Suppose that $\left\langle X_{\alpha}: \alpha<\alpha^{*}\right\rangle$ is a sequence of topological spaces and $\theta$ is an infinite cardinal. The $(<\theta)$-box topology on the product $\Pi_{\alpha<\alpha^{*}} X_{\alpha}$ has as a base, sets of the form $\Pi_{\alpha<\alpha^{*}} O_{\alpha}$, where each $O_{\alpha}$ is open in $X_{\alpha}$ and $\left|\left\{\alpha<\alpha^{*}: O_{\alpha} \neq X_{\alpha}\right\}\right|<\theta$.

So, in the case of $\theta=\aleph_{0}$, the $(<\theta)$-box topology is the usual Tychonoff topology. In the case of $\kappa=\aleph_{0}$, Observation 2.7 has another equivalent, which is that $X$ is compact if and only if every ultrafilter of closed subsets of $X$ has a nonempty intersection. The forward direction of this claim is clear, while for the backward direction, suppose that we have a filter $\mathcal{F}$ 
consisting of closed subsets of $\kappa$. Consider the collection $\mathcal{C}$ of all families of closed subsets of $X$ which contain $\mathcal{F}$ and which form a filter. Since $\mathcal{C}$ is nonempty, partially ordered by $\subseteq$ and closed under unions, by Zorn's lemma $\mathcal{C}$ has a maximal element $\mathcal{U}$, which is then an ultrafilter of closed sets. Hence $\bigcap \mathcal{U} \neq \emptyset$ and so $\bigcap \mathcal{F} \neq \emptyset$. However, if $\kappa>\aleph_{0}$, the notion of $(<\kappa)$-compactness does not have an ultrafilter characterisation. The above proof does not work since Zorn's lemma does not apply to the family of all $(<\kappa)$-complete filters of closed sets to produce a $(<\kappa)$-complete ultrafilter, but actually that analogue of Zorn's lemma leads to large cardinal notions, as we shall discuss below.

The ultrafilter equivalent of compactness is used in Tychonoff's proof that the Tychonoff product of compact spaces is compact and, for the reasons just explained, the analogue for the $(<\kappa)$-box topological product of $(<\kappa)$-compact spaces does not work. In fact, here is a well-known result for strongly compact cardinals.

Definition 2.9. A cardinal $\kappa$ is said to be $\lambda$-strongly compact if every $(<\kappa)$ complete filter $\mathcal{F}$ over any set $S$, generated by at most $\lambda$ many elements, can be extended to a $(<\kappa)$-complete ultrafilter over $S$.

$\kappa$ is strongly compact if it is $\lambda$-strongly compact for all $\lambda \geq \kappa$.

Theorem 2.10. The following statements are equivalent:

(1) The cardinal $\kappa$ is strongly compact;

(2) Any $(<\kappa)$-box product of $(<\kappa)$-compact spaces is $(<\kappa)$-compact;

(3) Any $(<\kappa)$-box product of discrete two-point spaces is $(<\kappa)$-compact.

Although this theorem is attributed to the mathematical folklore, we could not find a proof written in the literature. One of our main results, Theorem 5.1 below, is an elaboration of a similar characterisation but for weakly compact cardinals. A proof of Theorem 2.10 can be deduced by similar arguments as those that we used for the proof of Theorem 5.1.

\section{3. $\lambda$-Filter Extension Property}

Let us start with the following definition.

Definition 3.1. Suppose that $\kappa$ is an infinite cardinal and $\lambda \geq \kappa$. $\kappa$ is said to have the $\lambda$-filter extension property if for every family $\mathcal{A} \subseteq \mathcal{P}\left(2^{\lambda}\right)$ with $|\mathcal{A}| \leq \lambda$, every $(<\kappa)$-complete filter $\mathcal{F}$ over $2^{\lambda}$ generated by at most $\lambda$ many elements can be extended to a $(<\kappa)$-complete filter over $2^{\lambda}$ measuring $\mathcal{A}$. 
This section is devoted to the following Theorem 3.2, which will lead to an upper bound for the consistency strength of $\lambda$-square compact cardinals, as per Theorem 3.6.

Theorem 3.2. Suppose that $\lambda^{<\kappa}=\lambda$ for some infinite cardinals $\kappa$ and $\lambda$. Then the following are equivalent:

(1) $\kappa$ has the $\lambda$-filter extension property;

(2) Suppose that $\left\{X_{\gamma}: \gamma \leq \Gamma\right\}$ for some $\Gamma \leq \lambda$ is a family of $(<\kappa)$-compact spaces such that $w\left(X_{\gamma}\right) \leq \lambda$ for all $\gamma \in \Gamma$. Then the $(<\kappa)$-box product space $\prod_{\gamma \in \Gamma} X_{\gamma}$ is also $(<\kappa)$-compact;

(3) $2^{\lambda}$ is $(<\kappa)$-compact in the $(<\kappa)$-box topology.

To prove Theorem 3.2, we shall prove two lemmas.

Lemma 3.3. Suppose that $\lambda^{<\kappa}=\lambda$ and that $\kappa$ has the $\lambda$-filter extension property. Let $\Gamma \leq \lambda$ and suppose that $\left\{X_{\gamma}: \gamma \leq \Gamma\right\}$ is a family of $(<\kappa)$ compact spaces such that $w\left(X_{\gamma}\right) \leq \lambda$ for all $\gamma \in \Gamma$. Then the $(<\kappa)$-box product space $\prod_{\gamma \in \Gamma} X_{\gamma}$ is also $(<\kappa)$-compact.

Proof. Let $X$ denote the $(<\kappa)$-box product space $\prod_{\gamma \in \Gamma} X_{\gamma}$. Observe that $w(X) \leq \lambda$. Indeed, suppose that for each $\gamma$ we have a base $\mathcal{B}_{\gamma}$ for the topology on $X_{\gamma}$ with $\left|\mathcal{B}_{\gamma}\right| \leq \lambda$. We denote by $\pi_{\gamma}: X \rightarrow X_{\gamma}$, the projection of $X$ onto $X_{\gamma}$. Namely,

$$
\pi_{\gamma}(x)=x_{\gamma} \quad \text { for } x=\left\langle x_{\gamma}: \gamma \leq \Gamma\right\rangle .
$$

Then the base $\mathcal{B}$ (for the $(<\kappa)$-box topology) on $X$ is generated by taking $<\kappa$ intersections from the collection $\left\{\pi_{\gamma}^{-1}\left(U_{\gamma}\right): U_{\gamma} \in \mathcal{B}_{\gamma}, \gamma \in \Gamma\right\}$ is of cardinality $\leq \lambda$, by the cardinal arithmetic hypothesis on $\lambda$. It then follows that $X$ has size $\leq 2^{\lambda}$, so we can view $X$ as a subset of $2^{\lambda}$.

To show $(<\kappa)$-compactness of $X$, let $\mathcal{F}$ be a closed collection in $X$ satisfying the $(<\kappa)$-intersection property. One can also assume that for every $F \in \mathcal{F}, X \backslash F \in \mathcal{B}$. Since $|\mathcal{B}| \leq \lambda$, it is clear that $|\mathcal{F}| \leq \lambda$. Note that we can use $\lambda$-filter extension property of $\kappa$ on $|\mathcal{F}|$ since $X$ is a subset of $2^{\lambda}$. Therefore, since $|\mathcal{B}| \leq \lambda$, we can extend $\mathcal{F}$ to a $(<\kappa)$-complete filter $\mathcal{F}^{\prime}$ that measures $\mathcal{B}$. We claim that for each $\gamma \in \Gamma$

$$
\mathcal{F}_{\gamma}=\left\{\pi_{\gamma}(A): A \in \mathcal{F}^{\prime}\right\}
$$

is a $(<\kappa)$-complete filter in $X_{\gamma}$ that measures $\mathcal{B}_{\gamma}$. First it is clear that $\emptyset \notin \mathcal{F}_{\gamma}$. Secondly, if $B \supseteq \pi_{\gamma}(A)$ for some $A \in \mathcal{F}^{\prime}$, then $\pi_{\gamma}^{-1}(B) \supseteq A$, which implies $\pi_{\gamma}^{-1}(B) \in \mathcal{F}^{\prime}$. Hence

$$
B=\pi_{\gamma}\left(\pi_{\gamma}^{-1}(B)\right) \in \mathcal{F}_{\gamma} .
$$


Thirdly, if $B=\pi_{\gamma}(A), B^{\prime}=\pi_{\gamma}\left(A^{\prime}\right)$ for some $A, A^{\prime} \in \mathcal{F}^{\prime}$, then

$$
B \cap B^{\prime} \supseteq \pi_{\gamma}\left(A \cap A^{\prime}\right) .
$$

Since $A \cap A^{\prime} \in \mathcal{F}^{\prime}$, and hence $\pi_{\gamma}\left(A \cap A^{\prime}\right) \in \mathcal{F}_{\gamma}$, this implies (as has just been proved)

$$
B \cap B^{\prime} \in \mathcal{F}_{\gamma}
$$

The same is true for any collection $\left\{B_{\alpha}: \alpha \in I\right\}$ of size $<\kappa$ because $\mathcal{F}^{\prime}$ is $(<\kappa)$-complete. Therefore, $\mathcal{F}_{\gamma}$ is a $(<\kappa)$-complete filter of $X_{\gamma}$.

In fact $\mathcal{F}_{\gamma}$ measures $\mathcal{B}_{\gamma}$, for suppose $U_{\gamma} \in \mathcal{B}_{\gamma}$. Then $\pi_{\gamma}^{-1}\left(U_{\gamma}\right) \in \mathcal{B}$. Thus, either $\pi_{\gamma}^{-1}\left(U_{\gamma}\right)$ or $X \backslash \pi_{\gamma}^{-1}\left(U_{\gamma}\right)$ is in $\mathcal{F}^{\prime}$. In the first case, $U_{\gamma} \in \mathcal{F}_{\gamma}$, in the second case $X_{\gamma} \backslash U_{\gamma} \in \mathcal{F}_{\gamma}$, which is what we needed to show. Now $\overline{\mathcal{F}_{\gamma}}=$ $\left\{C: C \in \mathcal{F}_{\gamma}, C\right.$ is closed $\}$ is a closed collection in $X_{\gamma}$ satisfying the $(<$ $\kappa)$-intersection property, hence its intersection is nonempty. Suppose $x_{\gamma} \in$ $\bigcap \overline{\mathcal{F}_{\gamma}}$, then we can assert that

$$
x=\left\{x_{\gamma}: \gamma \in \Gamma\right\} \in \bigcap \mathcal{F} .
$$

To show this, suppose $U \in \mathcal{B}$ is a given basic neighbourhood of $x$. Then $U$ is of the form

$$
U=\prod_{i \in \Gamma_{0}} U_{\gamma_{i}} \times \prod\left\{X_{\gamma}: \gamma \neq \gamma_{i}, i \in \Gamma_{0}\right\}
$$

where each $U_{\gamma_{i}} \in \mathcal{B}_{\gamma_{i}}$ is a neighbourhood of $x_{\gamma_{i}}$ in $X_{\gamma_{i}}$, and $\Gamma_{0} \subseteq \Gamma$ has cardinality $<\kappa$. Note that $U$ can be expressed as

$$
U=\bigcap_{i \in \Gamma_{0}} \pi_{\gamma_{i}}^{-1}\left(U_{\gamma_{i}}\right)
$$

Now $\pi_{\gamma_{i}}^{-1}\left(U_{\gamma_{i}}\right) \in \mathcal{B}$. Suppose, however, that it is not in $\mathcal{F}^{\prime}$. Then $X \backslash$ $\pi_{\gamma_{i}}^{-1}\left(U_{\gamma_{i}}\right) \in \mathcal{F}^{\prime}$, since $\mathcal{F}^{\prime}$ measures $\mathcal{B}$. But then $X_{\gamma_{i}} \backslash U_{\gamma_{i}} \in \mathcal{F}_{\gamma_{i}}$, which is closed. This contradicts $x_{\gamma_{i}} \in \bigcap \overline{\mathcal{F}_{\gamma_{i}}}$. Consequently, we obtain $\pi_{\gamma_{i}}^{-1}\left(U_{\gamma_{i}}\right) \in \mathcal{F}^{\prime}$. Hence, in view of (1), we conclude that $U \in \mathcal{F}^{\prime}$ because $\mathcal{F}^{\prime}$ is $(<\kappa)$ complete. Therefore $x \in \bigcap \mathcal{F}$ and we can conclude that $X$ is a $(<\kappa)$ compact space.

The next lemma is motivated by the proof for the equivalence of the Ultrafilter Principle and Tychonoff's Theorem for compact Hausdorff spaces, see $[9,11]$.

Lemma 3.4. Suppose that $\kappa$ is an infinite cardinal and $\lambda^{<\kappa}=\lambda$. If $2^{\lambda}$ is $(<\kappa)$-compact in the $(<\kappa)$-box topology, then $\kappa$ has the $\lambda$-filter extension property.

Proof. If $\kappa=\aleph_{0}$, the conclusion follows from the Axiom of Choice. For $\kappa$ uncountable, we shall need the following observation. 
Sublemma 3.5. Suppose that $2^{\kappa}$ is $(<\kappa)$-compact in the $(<\kappa)$-box topology. Then we have that $2^{\theta}<\kappa$ whenever $\theta<\kappa$.

Proof. For each $f \in 2^{\theta}$ define $U_{f}=\left\{g \in 2^{\kappa}: f \subseteq g\right\}$, so an open set in the $(<\kappa)$-box topology of $2^{\kappa}$. It is not difficult to see $\left\{U_{f}: f \in 2^{\theta}\right\}$ forms a minimal open cover, so we have that its size satisfies $2^{\theta}<\kappa$ by the $(<\kappa)$-compactness of $2^{\kappa}$.

Now suppose that $\lambda \geq \kappa>\aleph_{0}$ satisfy the hypothesis of the lemma and let $\mathcal{F}_{0}$ be a $(<\kappa)$-complete filter on $2^{\lambda}$ which is generated by a family $\mathcal{E}$ of size $\leq \lambda$ and let $\mathcal{A}$ be a family of subsets of $2^{\lambda}$ of size $\leq \lambda$. We need to produce a $(<\kappa)$-complete filter $\mathcal{F}$ with $\mathcal{F}_{0} \subseteq \mathcal{F}$ that measures $\mathcal{A}$. For simplicity in notation, let us write $X$ for $2^{\lambda}$. The assumption that $2^{\lambda}$ is $(<\kappa)$-compact in the $(<\kappa)$-box topology implies that $2^{\kappa}$ is $(<\kappa)$-compact in the $(<\kappa)$-box topology, since $2^{\kappa}$ is homeomorphic to a closed subspace of $2^{\lambda}$. Therefore, by Sublemma 3.5, $2^{\theta}<\kappa$ whenever $\theta<\kappa$.

Let $\Sigma_{0}=\mathcal{E} \cup \mathcal{A} \cup \mathcal{A}^{c} \cup\{\emptyset\}$, where $\mathcal{C}^{c}=\{X \backslash S: S \in \mathcal{C}\}$ for any $\mathcal{C} \subseteq \mathcal{P}(X)$. Furthermore, let $\Sigma$ be the closure of $\Sigma_{0}$ under intersections of $<\kappa$ elements. Finally let $\Sigma^{\prime}=\Sigma \cup \Sigma^{c}$.

Consider $\mathcal{P}\left(\Sigma^{\prime}\right)$, identified with $2^{\Sigma^{\prime}}$. Note that $\left|\Sigma^{\prime}\right| \leq \lambda$ because $\lambda^{<\kappa}=\lambda$. Any $\mathcal{F} \in \mathcal{P}\left(\Sigma^{\prime}\right)$ is a collection of subsets of $X$.

Now let $2^{\Sigma^{\prime}}$ have the $(<\kappa)$-box product topology; then the $S^{\text {th }}$ coordinate projection $\pi_{S}: 2^{\Sigma^{\prime}} \rightarrow 2$ for any $S \in \Sigma^{\prime}$, defined by

$$
\pi_{S}(\mathcal{F})= \begin{cases}1 & \text { if } S \in \mathcal{F}, \\ 0 & \text { if } S \notin \mathcal{F},\end{cases}
$$

is continuous (in fact it is even continuous in the Tychonoff product topology), since for example $\pi_{S}^{-1}(\{1\})=\{\mathcal{F}: S \in \mathcal{F}\}$ is a basic clopen set. Let

$$
\begin{aligned}
D & =\left\{\mathcal{F} \in 2^{\Sigma^{\prime}}: \mathcal{F} \text { is a proper }(<\kappa) \text {-complete filter in } \Sigma^{\prime}\right\}, \\
E & =\left\{\mathcal{F} \in 2^{\Sigma^{\prime}}: \mathcal{F} \supseteq \mathcal{F}_{0}\right\} \text { and } \\
\Gamma_{S} & =\left\{\mathcal{F} \in 2^{\Sigma^{\prime}}: S \in \mathcal{F} \text { or } X \backslash S \in \mathcal{F}\right\}, \text { for each } S \in \Sigma^{\prime} .
\end{aligned}
$$

It is not difficult to see that

$$
\begin{aligned}
D & =\bigcap_{\substack{\Lambda \subseteq \Sigma^{\prime} \\
|\Lambda|<\kappa}}\left\{\mathcal{F} \in 2^{\Sigma^{\prime}}:\left(\left[(\forall A \in \Lambda) \pi_{A}(\mathcal{F})=1\right] \text { iff } \pi_{\cap \Lambda}(\mathcal{F})=1\right) \text { and } \pi_{\emptyset}(\mathcal{F})=0\right\} \\
E & =\bigcap_{A \in \Sigma^{\prime}}\left\{\mathcal{F} \in 2^{\Sigma^{\prime}}:\left[1-\pi_{A}(\mathcal{F})\right] \pi_{A}\left(\mathcal{F}_{0}\right)=0\right\}, \quad \text { and } \\
\Gamma_{S} & =\left\{\mathcal{F} \in 2^{\Sigma^{\prime}}: \pi_{S}(\mathcal{F})+\pi_{X \backslash S}(\mathcal{F}) \geq 1\right\} .
\end{aligned}
$$


We claim that by the continuity of the projections, the sets $D, E, \Gamma_{S}$ are closed. Let us check this for the set $D$.

We first note that for any $\Lambda \subseteq \Sigma^{\prime},|\Lambda|<\kappa$, the set

$$
O_{\Lambda}=\prod_{S \in \Sigma^{\prime}} U_{S}, \text { where } U_{S}= \begin{cases}\{0,1\} & \text { if } S \notin \Lambda, \\ \{1\} & \text { if } S \in \Lambda,\end{cases}
$$

is open in the $(<\kappa)$-box product topology. Consequently, $C_{\Lambda}=2^{\Sigma^{\prime}} \backslash O_{\Lambda}$ is closed. Now, for any such $\Lambda$,

$$
\left\{\mathcal{F} \in 2^{\Sigma^{\prime}}:\left(\left[(\forall A \in \Lambda) \pi_{A}(\mathcal{F})=1\right] \text { iff } \pi_{\cap \Lambda}(\mathcal{F})=1\right) \text { and } \pi_{\emptyset}(\mathcal{F})=0\right\}
$$

is equal to the set

$$
\left(C_{\Lambda} \cap \pi_{\cap \Lambda}^{-1}(\{0\}) \cap \pi_{\emptyset}^{-1}(\{0\})\right) \cup\left(\left(\bigcap_{A \in \Lambda} \pi_{A}^{-1}(\{1\})\right) \cap \pi_{\cap \Lambda}^{-1}(\{1\}) \cap \pi_{\emptyset}^{-1}(\{0\})\right),
$$

which is closed. Thus, taking the intersection over all $\Lambda \subseteq \Sigma^{\prime},|\Lambda|<\kappa$, we conclude that $D$ is closed.

It then follows that the set $\Phi_{S}=D \cap E \cap \Gamma_{S}$ is also closed, for any $S \in \Sigma^{\prime}$. Note that $\Phi_{S}$ is equal to the set

$\left\{\mathcal{F}: \mathcal{F}\right.$ is a proper $(<\kappa)$-complete filter in $\Sigma^{\prime}$ that includes $\mathcal{F}_{0}$ and contains at least one of $S$ or $X \backslash S\}$.

By Proposition 2.2, $\Phi_{S} \neq \emptyset$.

Recall the notation of grills from Definition 2.1 and let us now show that the collection of nonempty closed sets $\left\{\Phi_{S}: S \in\left(G\left(\mathcal{F}_{0}\right) \backslash \mathcal{F}_{0}\right) \cap \Sigma^{\prime}\right\}$ has the $(<\kappa)$-intersection property.

Consider a collection $\left\{\Phi_{S_{\alpha}}: \alpha \in \Lambda\right\}$, where $|\Lambda|<\kappa$. Take any $x \in X$ and for every $\alpha \in \Lambda$ choose $S_{\alpha}^{*} \in\left\{S_{\alpha}, X \backslash S_{\alpha}\right\}$ satisfying $x \in S_{\alpha}^{*}$. Then $x \in S^{*}=\bigcap_{\alpha \in \Lambda} S_{\alpha}^{*}$. Thus, by considering all possible intersections $\bigcap_{\alpha \in \Lambda} S_{\alpha}^{*}$ (there are $2^{|\Lambda|}<\kappa$ many, see the discussion at the beginning of the proof), where $S_{\alpha}^{*} \in\left\{S_{\alpha}, X \backslash S_{\alpha}\right\}$, we form a partition of $X$ of cardinality $<\kappa$. Since $X \in G\left(\mathcal{F}_{0}\right) \cap \Sigma^{\prime}$ it follows that one of the intersections of the form $\bigcap_{\alpha \in \Lambda} S_{\alpha}^{*}$ must be in $G\left(\mathcal{F}_{0}\right) \cap \Sigma^{\prime}$ by property (3) of the grills. Let $S^{\prime}=$ $\bigcap_{\alpha \in \Lambda} S_{\alpha}^{*} \in G\left(\mathcal{F}_{0}\right) \cap \Sigma^{\prime}$. Note that $S^{\prime} \notin \mathcal{F}_{0}$, otherwise $S_{\alpha}^{*} \in \mathcal{F}_{0}$ and these sets are taken from $G\left(\mathcal{F}_{0}\right) \backslash \mathcal{F}_{0}$. Consequently, $S^{\prime} \in G\left(\mathcal{F}_{0}\right) \backslash \mathcal{F}_{0}$ and therefore $X \backslash S^{\prime} \in G\left(\mathcal{F}_{0}\right) \backslash \mathcal{F}_{0}$. It then follows, again by applying Proposition 2.2 , that there exists a $(<\kappa)$-complete filter $\mathcal{G}$ in $\Sigma^{\prime}$ that extends $\mathcal{F}_{0}$ and contains $S^{\prime}$. Then $\mathcal{G}$ is in the intersection $\bigcap_{\alpha \in \Lambda} \Phi_{S_{\alpha}}$, which is therefore nonempty, as required.

By our assumption, $2^{\Sigma^{\prime}}$ is $(<\kappa)$-compact; hence there exists some $\mathcal{U}$ in the intersection $\bigcap_{S \in\left(G\left(\mathcal{F}_{0}\right) \backslash \mathcal{F}_{0}\right) \cap \Sigma^{\prime}} \Phi_{S}$. Then $\mathcal{U}$ is a $(<\kappa)$-complete filter on 
$\Sigma^{\prime}$ that extends $\mathcal{F}_{0}$ and measures $\mathcal{A}$. One can then extend $\mathcal{U}$ to a $(<\kappa)$ complete filter on $\mathcal{P}(X)$ by taking supersets of sets from $\mathcal{U}$.

Proof of Theorem 3.2. The implication (1) $\Longrightarrow(2)$ is given by Lemma 3.3. The implication $(2) \Longrightarrow(3)$ is obvious. The implication $(3) \Longrightarrow(1)$ is given by Lemma 3.4.

Using the same argument as in the proof of Lemma 3.3 we obtain:

Theorem 3.6. For any cardinals $\kappa \leq \lambda$, if $\kappa$ has the $\lambda$-filter extension property, then $\kappa$ is $\lambda$-square compact.

Of course, a direct consequence of Definitions 2.9 and 3.1 is

Proposition 3.7. If $\kappa$ is $\lambda$-strongly compact then it has the $\lambda$-filter extension property.

We do not know if the converse of Theorem 3.6 holds, leading to an open question:

Problem 3.8. If a cardinal $\kappa$ is $\lambda$-square compact, does it necessarily have the $\lambda$-filter extension property?

\section{Embedding Characterisations of the $\lambda$-Filter extension PROPERTY}

Let us recall the following characterisation of $\lambda$-strongly compact cardinals, ([5, Th. 22.17, Pg. 307]):

- A cardinal $\kappa$ is $\lambda$-strongly compact if and only if there exists an elementary embedding $j: V \rightarrow M$ with critical point $\kappa$, such that for all $X \subseteq M$ with $|X| \leq \lambda$, there exists $Y \in M$ with $X \subseteq Y$ and $M \models|Y|<j(\kappa)$.

We shall prove that the $\lambda$-filter extension property is equivalent to a "mini" version of the above characterisation of $\lambda$-strongly compact cardinals. That mini version has in fact appeared in the recent literature, it was introduced by P. A. White in [12], under the name nearly $\lambda$-strongly compact cardinals.

Definition 4.1. The cardinal $\kappa$ is said to be nearly $\lambda$-strongly compact if for every family $\mathcal{A}$ of $\lambda$-many subsets of $\mathcal{P}_{\kappa}(\lambda)$ there is a non-principal $(<\kappa)$-complete fine filter $\mathcal{F}$ over $\mathcal{P}_{\kappa}(\lambda)$ measuring $\mathcal{A}$.

Theorem 4.2. Suppose that $\kappa>\aleph_{0}$ and $\lambda^{<\kappa}=\lambda$. Then:

- $\kappa$ has the $\lambda$-filter extension property, if and only if 
- $\kappa$ is nearly $\lambda$-strongly compact, if and only if

any of the following three statements hold:

(A) For every large enough regular cardinal $\chi$, every model $M \prec H(\chi)$ with $|M|=\lambda, \lambda \subseteq M$ and $^{\kappa>} M \subseteq M$, there exists an elementary embedding $j: M \rightarrow N$ with critical point $\kappa$, such that for all $X \subseteq N$ with $|X| \leq \lambda$, there exists $Y \in N$ with $X \subseteq Y$ and $N \models|Y|<j(\kappa)$.

(B) For every large enough regular cardinal $\chi$, for every $T \in H(\chi)$, there is a model $M \prec H(\chi)$ satisfying $T \in M,|M|=\lambda, \lambda \subseteq M$ and $^{\kappa>} M \subseteq M$, with an elementary embedding $j: M \rightarrow N$ having critical point $\kappa$, such that for all $X \subseteq N$ with $|X| \leq \lambda$, there exists $Y \in N$ with $X \subseteq Y$ and $N \models|Y|<j(\kappa)$.

(C) For every $A \subseteq \lambda$, there is a transitive $M \models Z F C^{-}$with $\kappa, \lambda \in M$, $A \subseteq M,|M|=\lambda,{ }^{\kappa>} M \subseteq M$, and an $N$ with an elementary embedding $j: M \rightarrow N$ having critical point $\kappa$, such that $j(\kappa)>\lambda$ and there is an $s \in N$ such that $j^{\prime \prime}(\lambda) \subseteq s$ and $|s|^{N}<j(\kappa)$.

We prove the theorem through a series of lemmas, assuming throughout that $\kappa$ is uncountable and $\lambda^{<\kappa}=\lambda$.

Lemma 4.3. $(A) \Longrightarrow$ ( $\kappa$ has the $\lambda$-filter extension property) $\Longrightarrow$ ( $\kappa$ is nearly $\lambda$-strongly compact) $\Longrightarrow(A)$.

Proof of $(\mathrm{A}) \Longrightarrow(\kappa$ has the $\lambda$-filter extension property $)$. Let $\mathcal{F}$ be a $(<$ $\kappa)$-complete filter on $2^{\lambda}$ which is generated by a family $\mathcal{E}$ of size $|\mathcal{E}| \leq \lambda$ and let $\mathcal{A}$ be a family of subsets of $2^{\lambda}$ with $|\mathcal{A}| \leq \lambda$. Let $\chi=\left(2^{\lambda}\right)^{+}$, so $\mathcal{F}, \mathcal{E}, \mathcal{A} \in H(\chi)$. Let $M \prec H(\chi)$ be a model with $\mathcal{E}, \mathcal{F}, \lambda \in M,|M|=\lambda$, $\lambda \cup \mathcal{A} \cup \mathcal{E} \subseteq M$ and ${ }^{\kappa>} M \subseteq M$. Furthermore, let $j: M \rightarrow N$ be an elementary embedding with critical point $\kappa$ as in $(\mathrm{A})$. For $X:=j^{\prime \prime}(\mathcal{F} \cap M)$ there exists $Y \in N$ with $X \subseteq Y$ and $N \models|Y|<j(\kappa)$.

By elementarity of the embedding $j, j(\mathcal{F})$ is a $(<j(\kappa))$-complete filter of subsets of $j\left(2^{\lambda}\right)$. Now $j(\mathcal{F}) \cap Y \subseteq \mathcal{P}\left(j\left(2^{\lambda}\right)\right)$ and $|j(\mathcal{F}) \cap Y|<j(\kappa)$. Consequently, $N \models \bigcap(j(\mathcal{F}) \cap Y) \neq \emptyset$, and therefore there exists some $c \in N \cap(\bigcap(j(\mathcal{F}) \cap Y))$. We shall use $c$ to define a filter $\mathcal{F}^{*}$ on $2^{\lambda}$ with $\mathcal{F} \subseteq \mathcal{F}^{*}$ and such that all sets in $\mathcal{A}$ are measured by $\mathcal{F}^{*}$.

To define $\mathcal{F}^{*}$ we first define an $M$-ultrafilter $\mathcal{F}^{\prime}$, that is a family $\mathcal{F}^{\prime} \subseteq$ $\mathcal{P}\left(2^{\lambda}\right)$ such that $\mathcal{F}^{\prime}$ is a filter and it measures every element of $\mathcal{P}\left(2^{\lambda}\right) \cap M$. For $A \in \mathcal{P}\left(2^{\lambda}\right) \cap M$, we let

$$
A \in \mathcal{F}^{\prime} \Longleftrightarrow c \in j(A) .
$$

- If $A \in \mathcal{F} \cap M$ then $j(A) \in j^{\prime \prime}(\mathcal{F} \cap M)=X \subseteq Y$. Moreover, it follows from elementarity of $j$, that $j(A) \in j(\mathcal{F})$ since $A \in \mathcal{F}$. Thus, 
$j(A) \in j(\mathcal{F}) \cap Y$ and therefore, $c \in j(A)$. We have shown that $\mathcal{F} \cap M \subseteq \mathcal{F}^{\prime}$. Since $\mathcal{E} \subseteq M \cap \mathcal{F}$ and $\mathcal{E}$ generates $\mathcal{F}$, it follows that in fact $\mathcal{F} \subseteq \mathcal{F}^{\prime}$.

- Let us show that $\mathcal{F}^{\prime}$ is $(<\kappa)$-complete: Given a family $\left\{A_{i}: i<i^{*}<\right.$ $\kappa\} \subseteq \mathcal{F}^{\prime}$, we have

$$
j\left(\bigcap_{i<i^{*}} A_{i}\right)=\bigcap_{i<i^{*}} j\left(A_{i}\right) \ni c .
$$

Consequently, $\bigcap_{i<i^{*}} A_{i} \in \mathcal{F}^{\prime}$. Note that $\left\{A_{i}: i<i^{*}\right\} \in M$ since ${ }^{\kappa>} M \subseteq M$.

- We next show that $\mathcal{F}^{\prime}$ is an $M$-ultrafilter on $2^{\lambda}$. Indeed, if $A \in$ $\mathcal{P}\left(2^{\lambda}\right) \cap M$, then $c \in j(A)$ or $c \in j\left(2^{\lambda}\right) \backslash j(A)=j\left(2^{\lambda} \backslash A\right)$.

Since $\mathcal{A} \subseteq \mathcal{P}\left(2^{\lambda}\right) \cap M$, we have that $\mathcal{F}^{\prime}$ measures all of $\mathcal{A}$. We then use $\mathcal{F}^{\prime}$ to generate (in $V$ ) a $(<\kappa)$-complete filter $\mathcal{F}^{*}$ on $2^{\lambda}$ by letting for $A \subseteq 2^{\lambda}$,

$$
A \in \mathcal{F}^{*} \Longleftrightarrow A \supseteq B \text { for some } B \in \mathcal{F}^{\prime} .
$$

It is standard to verify that $\mathcal{F}^{*}$ is as required.

Proof of ( $\lambda$-filter extension property) $\Longrightarrow$ (nearly $\lambda$-strongly compact).

Let $\kappa$ be a cardinal with the $\lambda$-filter extension property. Note that by our assumptions $\left|\mathcal{P}_{\kappa}(\lambda)\right|=\lambda$, so using any fixed bijection between $\mathcal{P}_{\kappa}(\lambda)$ and $\lambda$, we can treat filters on $\mathcal{P}_{\kappa}(\lambda)$ as if they were filters on $\lambda$. Note also that the $\lambda$-filter extension property of $\kappa$ implies that every $(<\kappa)$-complete filter $\mathcal{F}_{0}$ on $\lambda$ generated by a family $\mathcal{E}$ consisting of $\leq \lambda$ sets, can be extended to a $(<\kappa)$-complete filter on $\lambda$ which measures a given family $\mathcal{A}$ of $\leq \lambda$ subsets of $\lambda$. Namely, we shall consider $\lambda$ and $2^{\lambda}$ as ordinals where $\lambda<2^{\lambda}$. Then we can define $\mathcal{F}_{0}^{+}=\left\{B \subseteq 2^{\lambda}: B \cap \lambda \in \mathcal{F}_{0}\right\}$. This is clearly a $(<\kappa)$-complete filter on $2^{\lambda}$ and is generated by $\mathcal{E}$. By the $\lambda$-filter extension property of $\kappa$, there is a $(<\kappa)$-complete filter $\mathcal{F}^{+}$on $2^{\lambda}$ which extends $\mathcal{F}_{0}^{+}$and measures $\mathcal{A}$. Let $\mathcal{F}=\left\{B \cap \lambda: B \in \mathcal{F}^{+}\right\}$. Clearly $\mathcal{F}_{0} \subseteq \mathcal{F}$. Since $\lambda \in \mathcal{F}_{0}$, we have $\lambda \in \mathcal{F}_{0}^{+}$ and so $\lambda \in \mathcal{F}^{+}$. This suffices to prove that the $(<\kappa)$-completeness of $\mathcal{F}^{+}$ implies the $(<\kappa)$-completeness of $\mathcal{F}$. Finally, it is clear that $\mathcal{F}$ measures $\mathcal{A}$.

Consider a family $\mathcal{A}$ of $\lambda$-many subsets of $\mathcal{P}_{\kappa}(\lambda)$ and let $B_{\alpha}=\{a \in$ $\left.\mathcal{P}_{\kappa}(\lambda): \alpha \in a\right\} \subseteq \mathcal{P}_{\kappa}(\lambda)$ for every $\alpha<\lambda$. Let $\mathcal{F}_{0}$ be the filter on $\mathcal{P}_{\kappa}(\lambda)$ generated by the family $\mathcal{B}=\left\{B_{\alpha}: \alpha<\lambda\right\}$, which is of size at most $\lambda$. Then $\mathcal{F}_{0}$ is $(<\kappa)$-complete. By the above remarks, the $\lambda$-filter extension property of $\kappa$ implies that there is a $(<\kappa)$-complete filter $\mathcal{F}$ on $\mathcal{P}_{\kappa}(\lambda)$ with $\mathcal{F}_{0} \subseteq \mathcal{F}$ that measures $\mathcal{A}$. The filter $\mathcal{F}$ is fine because each $B_{\alpha}$ is already in $\mathcal{F}_{0}$. Finally, it is non-principal, since if $\bigcap \mathcal{F} \neq \emptyset$, then $\bigcap \mathcal{F}_{0} \neq \emptyset$ and in 
particular there is $a \in \mathcal{P}_{\kappa}(\lambda)$ with $a \in \bigcap_{\alpha<\lambda} B_{\alpha}$. Hence $\lambda \subseteq a$, which is impossible since $a \in \mathcal{P}_{\kappa}(\lambda)$ and $\lambda \geq \kappa$.

Proof of ( $\kappa$ is nearly $\lambda$-strongly compact) $\Longrightarrow(\mathrm{A})$. Let $\chi$ and $M$ be as in (A). Note that such an $M$ exists since $\lambda^{<\kappa}=\lambda$. Let $S=\mathcal{P}_{\kappa}(\lambda)$. Since $\lambda \subseteq M$ and ${ }^{\kappa>} M \subseteq M$, we have $\mathcal{P}_{\kappa}(\lambda) \subseteq M$. For $a \in S$, let $C_{a}=\{b \in$ $\left.\mathcal{P}_{\kappa}(\lambda): a \subseteq b\right\}$ and $\mathcal{E}=\left\{C_{a}: a \in \mathcal{P}_{\kappa}(\lambda)\right\}$. Next, let $\mathcal{F}$ be the non-principal $(<\kappa)$-complete fine filter on $S$ measuring every element of $\mathcal{P}(S) \cap M$, which exists by our assumption. Note that $\mathcal{F} \supseteq \mathcal{E}$ and is an $M$-ultrafilter on $S$.

We now form a subset of the ultrapower $M^{S} / \mathcal{F}$, where we consider only those functions $f: S \rightarrow M$ that are in $M$, which we denote by $\left(M^{S} / \mathcal{F}\right)_{M}$. Since $\mathcal{F}$ decides every element of $\mathcal{P}(S) \cap M,\left(M^{S} / \mathcal{F}\right)_{M}$ is an $M$-ultrapower, that is the relations $f={ }_{\mathcal{F}} g$ and $f \in_{\mathcal{F}} g$ are still equivalence relations, as the sets $\{a: f(a)=g(a)\}$ and $\{a: f(a) \in g(a)\}$ are both in $M$ if $f, g \in M$.

Since $\kappa$ is uncountable and $\mathcal{F}$ is $(<\kappa)$-complete, it is closed under intersections of length $\leq \omega$. Therefore we one can prove in a standard way that there cannot be an infinite decreasing sequence

$$
\left[a_{0}\right]_{\mathcal{F}} \ni\left[a_{1}\right]_{\mathcal{F}} \ni \cdots \ni\left[a_{n}\right]_{\mathcal{F}} \ni \ldots
$$

of elements of $\left(M^{S} / \mathcal{F}\right)_{M}$, see for example [5, Prop. 5.3, Pg. 48]. Hence, $\left(M^{S} / \mathcal{F}\right)_{M}$ is well-founded.

Let $N=\left(M^{S} / \mathcal{F}\right)_{M}$ and define $j: M \rightarrow N$, with $j(a)=[a]_{\mathcal{F}}$. It follows that $j$ is an elementary embedding.

Next, let $X=\left\{\left[f_{\alpha}\right]_{\mathcal{F}}: \alpha<\lambda\right\}$ be a subset of $N$. Define $F: \mathcal{P}_{\kappa}(\lambda) \rightarrow M$, by $F(a)=\left\{f_{\alpha}(a): \alpha \in a\right\}$, which is $\in M$ since $^{\kappa>} M \subseteq M$. Put $Y=[F]_{\mathcal{F}} \in$ $N$ and we shall show that $X \subseteq Y$. We need to show that for every $\alpha<\lambda$ we have $\left[f_{\alpha}\right]_{\mathcal{F}} \in[F]_{\mathcal{F}}$, i.e. that $\left\{a: f_{\alpha}(a) \in F(a)\right\} \in \mathcal{F}$. But

$$
\left\{a: f_{\alpha}(a) \in F(a)\right\}=\{a: \alpha \in a\}=C_{\{\alpha\}} \in \mathcal{F}_{0} .
$$

To see that $N \models|Y|<j(\kappa)$, note that $|F(a)| \leq|a|<\kappa$ for each $a \in S$, so that $N \models\left|[F]_{\mathcal{F}}\right|<j(\kappa)$.

Finally, observe that $j(\alpha)=\alpha$ for $\alpha<\kappa$. Indeed, if not, let $\alpha<\kappa$ be the least such that $j(\alpha)>\alpha$. If $j(\alpha)>[f]_{\mathcal{F}}=\alpha$, then $\{a \in S$ : $f(a)<\alpha\} \in \mathcal{F}$, and so by $(<\kappa)$-completeness there is a $\beta<\alpha$ such that $\{a \in S: f(a)=\beta\} \in \mathcal{F}$. But then, $[f]_{\mathcal{F}}=j(\beta)=\beta<\alpha$, a contradiction. Taking $X=\left\{[\alpha]_{\mathcal{F}}: \alpha<\kappa\right\}$ in the argument of the preceeding paragraph, we see that $\kappa<j(\kappa)$ so that $j$ has critical point $\kappa$.

Lemma 4.4. $(A) \Longrightarrow(B) \Longrightarrow$ ( $(\kappa$ has the $\lambda$-filter extension property). 
Proof of (A) $\Longrightarrow(\mathrm{B})$. Let us fix any $M \prec H(\chi)$ satisfying $T \in M,|M|=$ $\lambda, \lambda \subseteq M$ and ${ }^{\kappa>} M \subseteq M$. This is possible because $T \in H(\chi)$. We then apply (A) to obtain (B).

Proof of $(\mathrm{B}) \Longrightarrow\left(\kappa\right.$ has the $\lambda$-filter extension property). Let $\mathcal{F}_{0}, \mathcal{E}$ and $\mathcal{A}$ be as in the definition of the $\lambda$-filter extension property and let

$$
T=\left\{\mathcal{E}, \mathcal{F}_{0}, \mathcal{A}, \lambda, 2^{\lambda}\right\} .
$$

Let $\chi=\left(2^{\lambda}\right)^{+}$. As guaranteed by (B), we can choose $M \prec H(\chi)$ satisfying $T \in M,|M|=\lambda, \lambda \subseteq M$ and ${ }^{\kappa>} M \subseteq M$ and an embedding $j$ satisfying the properties stated in (B). Since $|\mathcal{A}|=\lambda$ and $\mathcal{A}, \lambda \in M$, there is a bijection $f: \lambda \rightarrow \mathcal{A}$ with $f \in M$ (by elementarity). Hence, for every $\alpha<\lambda, f(\alpha) \in M$ and in particular, $\mathcal{A}=\{f(\alpha): \alpha<\lambda\} \subseteq M$. Similarly for $\mathcal{E}$.

Hence, $M$ and $j$ satisfy the assumptions of the proof of $(\mathrm{A}) \Longrightarrow(\kappa$ has the $\lambda$-filter extension property) above and the same proof gives us that $\kappa$ has the $\lambda$-filter extension property holds.

Proof of Theorem 4.2. It is proved in [12], by an argument credited to Cody and White, that $(\mathrm{C})$ is equivalent to $\kappa$ being nearly $\lambda$-strongly compact. Together with the results of Lemma 4.3 and Lemma 4.4, this proves the theorem.

Remark 4.5. Note that in the proof of $(\mathrm{A}) \Longrightarrow(\kappa$ has the $\lambda$-filter extension property) in Lemma 4.3, the $(<\kappa)$-complete filter $\mathcal{F}$ could have been taken on any set $S$ instead of $2^{\lambda}$. This shows that Definition 3.1 can be restated as:

- For every set $S$ and every family $\mathcal{A} \subseteq \mathcal{P}(S)$ with $|\mathcal{A}| \leq \lambda$, every $(<\kappa)$-complete filter $\mathcal{F}$ over $S$ generated by at most $\lambda$ many elements can be extended to a $(<\kappa)$-complete filter over $S$ measuring $\mathcal{A}$.

\section{Weakly Compact Cardinals}

The following Theorem 5.1 gives several characterisations of weakly compact cardinals in terms of productivity of topological spaces. This theorem, which we believe is of independent interest, will be used in Section 6 to give a lower bound for the consistency strength of the notion of $\lambda$-square compactness. Many parts of this theorem are known but not always easy to find in the literature. We in particular point out the direct proof of $(1) \Longleftrightarrow(3)$ by Luca Motto Ros in [8], while a more general statement relative to $(1) \Longleftrightarrow(3)$ and further references, some of them quite old, can be found in [7]. 
Theorem 5.1. Suppose that $\kappa=\kappa^{<\kappa}>\aleph_{0}$. Then the following are equivalent:

(1) $\kappa$ is a weakly compact cardinal;

(2) Suppose that $\left\{X_{\gamma}: \gamma \leq \Gamma\right\}$ for some $\Gamma \leq \kappa$ is a family of $(<\kappa)$-compact spaces such that $w\left(X_{\gamma}\right) \leq \kappa$ for all $\gamma \in \Gamma$. Then the $(<\kappa)$-box product space $\prod_{\gamma \in \Gamma} X_{\gamma}$ is also $(<\kappa)$-compact;

(3) $2^{\kappa}$ is $(<\kappa)$-compact in the $(<\kappa)$-box topology;

(4) $\kappa$ has the $\kappa$-filter extension property;

(5) Suppose that $\left\{X_{\gamma}: \gamma \leq \Gamma\right\}$ for some $\Gamma \leq \kappa$ is a family of $(<\kappa)$-compact spaces such that $w\left(X_{\gamma}\right) \leq \kappa$ for all $\gamma \in \Gamma$. Then the Tychonoff product space $\prod_{\gamma \in \Gamma} X_{\gamma}$ is also $(<\kappa)$-compact;

(6) $\kappa$ is a weakly square compact cardinal;

(7) The product of any two $(<\kappa)$-compact GO-spaces of weight $\leq \kappa$ is $(<\kappa)$-compact.

We recall that a generalised ordered space (GO-space) is a Hausdorff space equipped with a linear order and having a base of order-convex sets.

We also recall that a cardinal $\kappa$ is said to have the tree property if every tree of cardinality $\kappa$ whose every level has cardinality $<\kappa$, has a branch of cardinality $\kappa$. It is well known that $\kappa$ is weakly compact if and only if it is strongly inaccessible and has the tree property, see [5].

Many parts of Theorem 5.1 follow from what has already been proved. Once we prove the following Lemma 5.2, we shall be ready for the proof of Theorem 5.1.

Lemma 5.2. If a cardinal $\kappa$ is weakly compact then $2^{\kappa}$ is $(<\kappa)$-compact in the $(<\kappa)$-box topology.

Proof. One can note that $2^{\kappa}$ can be viewed as a tree $T$ of height $\kappa$ such that each level has cardinality $<\kappa$, since $\kappa$ is a strong limit. Note that branches are closed sets in the $(<\kappa)$-box topology described below.

Suppose that $\mathcal{F}$ is a $(<\kappa)$-complete filter of closed sets in $2^{\kappa}$ equipped with the $(<\kappa)$-box topology. We define a tree $\hat{T}$ in the following manner: an element $s \in 2^{\beta}$, where $\beta<\kappa$, is in $\hat{T}$ if for every $F \in \mathcal{F}$ there is a branch $b \in F$ such that $b \uparrow \beta=s$. In other words, the open set $U_{s}$ intersects $F$ for every $F \in \mathcal{F}$, where $U_{s}=\prod_{\alpha<\kappa} V_{\alpha}$ with $V_{\alpha}=\{s(\alpha)\}$ for $\alpha<\beta$ and $V_{\alpha}=\{0,1\}$ for $\beta \leq \alpha<\kappa$.

Let us show that for any $\beta<\kappa, \hat{T}$ has nodes on the $\beta^{\text {th }}$ level. Suppose that for any $s \in 2^{\beta}, s \notin \hat{T}$. Then for any $s \in 2^{\beta}$ there exists an $F_{s} \in \mathcal{F}$ such that $F_{s} \cap U_{s}=\emptyset$. Now $2^{\beta}<\kappa$ and $\mathcal{F}$ is $(<\kappa)$-complete so that $F=$ 
$\bigcap_{s \in 2^{\beta}} F_{s} \in \mathcal{F}$. However, $U=\bigcup_{s \in 2^{\beta}} U_{s}=2^{\kappa}$ and $F \cap U=\emptyset$, contradicting $F \neq \emptyset$.

Thus, $\hat{T}$ is a $\kappa$-tree. By the tree property, there is a $\kappa$-branch $b \in[\hat{T}]$ and by definition of $\hat{T}$ it follows that $b \in F$ for every $F \in \mathcal{F}$. Indeed, if $b \notin F$ for some $F \in \mathcal{F}$, then there is some $\beta<\kappa$ and an open neighbourhood $U_{b}$ of $b$ of the form $\prod_{\alpha<\kappa} V_{\alpha}$ with $V_{\alpha}=\{b(\alpha)\}$ for $\alpha<\beta$ and $V_{\alpha}=\{0,1\}$ for $\beta \leq \alpha<\kappa$, satisfying $U_{b} \cap F=\emptyset$. But $s=b\left\lceil\beta \in \hat{T}\right.$, so that $U_{s}=U_{b}$ intersects every $F \in \mathcal{F}$, a contradiction. Consequently, $\cap \mathcal{F} \neq \emptyset$.

We can now give a proof of Theorem 5.1.

Proof of Theorem 5.1. (1) $\Longrightarrow$ (3) is the content of Lemma 5.2. Therefore (2), (3) and (4) are equivalent by Theorem 3.2.

Implications $(2) \Longrightarrow(5) \Longrightarrow(6) \Longrightarrow(7)$ are obvious.

We are only left with $(7) \Longrightarrow(1)$. This follows from the argument in the paper of Hajnal and Juhász [2], which we recall as it is not phrased in these terms in the original paper. By a result of Hanf in [3], if $\kappa$ is not weakly compact then there exists a linearly ordered set $(L, \leq)$ with $|L| \geq \kappa$, in which every decreasing or increasing well-ordered (by $\leq$ ) subset is of cardinality $<\kappa$. By taking a suborder if necessary, we may assume that $|L|=\kappa$. To get the counterexample in [2], one has to assume that $(L, \leq)$ is continuously ordered, that is for all non-empty $A, B \subseteq L$, if $A<B$, then there is $c$ such that $A<c<B$, where we write $A<B$ for subsets of $L$ if $a<b$ for all $a \in A$ and $b \in B$, a property which can be obtained in a standard way by an inductive construction in which all the gaps $(A, B)$ are filled. This procedure (which leads to what is called a linear compactification $c L$ of the original $L$ ) may in general increase the size of $L$ but in our particular case it does not, since $\kappa^{<\kappa}=\kappa$. The linear compactification will preserve the property that every decreasing or increasing well-ordered subset is of cardinality $<\kappa$. So we shall assume that $L$ is already continuous.

To pass to the topological spaces, we take two copies of $L$, which we denote by $X_{r}$ and $X_{l}$. The basic open sets are half-open intervals of the form $(x, y]$ for $X_{r}$ and the ones of the form $[x, y)$ for $X_{l}$. This makes $X_{l}$ and $X_{r}$ GO spaces. Both of these spaces have size and so weight $\leq \kappa$. It is shown in [2] that both $X_{l}$ and $X_{r}$ are $(<\kappa)$-compact, while their product $X_{l} \times X_{r}$ is not.

\section{Consistency Strength of $2^{\kappa}$-SQuare COMPaCtness}

As mentioned above, [2] proves that a fully square compact cardinal must be weakly compact and it is clear from the definition and the equivalences 
listed in Theorem 2.10 that a strongly compact cardinal is square compact. We shall consider the consistency strength of $\lambda$-square compactness, getting the same lower bound of weak compactness, but a much better upper bound than strong compactness.

Our main findings can be summarised as follows. We remind the reader that the definition implies that being $\lambda$-square compact is a notion that increases in strength with $\lambda$.

Theorem 6.1. Suppose that $\kappa$ is uncountable. Then

(1) If $\kappa=\kappa^{<\kappa}$ is $\lambda$-square compact for any $\lambda \geq \kappa$, then $\kappa$ is weakly compact.

(2) It is consistent modulo large cardinals that the first weakly compact cardinal $\kappa$ is $2^{\kappa}$-square compact.

Item (2) above implies that from $\kappa$ being $2^{\kappa}$-square compact, one cannot infer that $\kappa$ has any large cardinal strength larger than weak compactness. Namely:

Corollary 6.2. Suppose that $\varphi$ is a large cardinal property such that any $\kappa$ satisfying $\varphi(\kappa)$ has an unbounded set of weakly compact cardinals below. Then, it is consistent modulo large cardinals that there is a $\kappa$ which is $2^{\kappa}$ square compact, but it does not have property $\varphi$.

We recall that being strongly compact, being measurable or even just being indescribable are large notions such that any cardinal satisfying them has an unbounded set of weakly compact cardinals below. See [5].

Proof of Theorem 6.1. (1) follows from the Theorem 5.1. For item (2), we shall need to refer to the notion of nearly $\lambda$-supercompactness, defined by Jason Schanker in [10]. In the case of $\lambda=\lambda^{<\kappa}$, Theorem 1.4. of [10] gives that $\kappa$ is nearly $\lambda$-supercompact if and only if for every family $\mathcal{A}$ of $\lambda$-many subsets of $\mathcal{P}_{\kappa}(\lambda)$ and a collection $\mathcal{G}$ of $\lambda$-many functions from $\mathcal{P}_{\kappa}(\lambda)$ to $\lambda$, there is a non-principal $(<\kappa)$-complete fine $\mathcal{G}$-normal filter $\mathcal{F}$ over $\mathcal{P}_{\kappa}(\lambda)$ which measures $\mathcal{A}$.

It is then evident from Definition 4.1 that in the case of $\lambda=\lambda^{<\kappa}$, every nearly $\lambda$-supercompact cardinal is nearly $\lambda$-strongly compact and therefore by Theorem $4.2, \kappa$ has the $\lambda$-filter extension property.

Now let us place ourselves in any universe of set theory in which some cardinal $\kappa$ is nearly $\lambda$-supercompact for some $\lambda=\lambda^{<\kappa}$. An example of such a universe is one in which $\kappa$ is a supercompact cardinal and $\lambda=2^{\kappa}$, since any cardinal which is $\lambda$-supercompact is nearly $\lambda$-supercompact (see Observation 1.2 (1) of [10]). Theorem 7 of [1] gives a forcing extension of 
such a universe in which $2^{\kappa}=\lambda$ and $\kappa$ is simultaneously the first weakly compact cardinal and nearly $\lambda$-supercompact. In particular $\kappa$ is nearly $2^{\kappa}$ strongly compact, has the $2^{\kappa}$-filter extension property and, by Theorem 4.2 is $2^{\kappa}$-square compact.

It is of course interesting to ask if in Theorem 6.1 we can say something about $\lambda$-square compactness for $\lambda>2^{\kappa}$. The methods of [1] cannot help us here, since they necessarily make $2^{\kappa}=\lambda$. There is another way for obtaining near $\lambda$-super compactness without measurability, as in [10], but as proved in the same paper, this method is limited to $\lambda \leq \kappa^{+}$.

We cannot hope to use nearly $\lambda$-strong compactness to show that the fully square compact cardinals have low consistency strength, as can be seen by the following theorem.

Theorem 6.3. Suppose that $\kappa$ is nearly $\lambda$-strongly compact for unboundedly many $\lambda$. Then $\kappa$ is strongly compact.

Proof. Indeed, from what is proved above, it follows that in such a case, $2^{\lambda}$ is $(<\kappa)$-compact in the $(<\kappa)$-box topology for unboundedly many $\lambda$. Consequently, any $(<\kappa)$-box product of discrete two-point spaces is $(<\kappa)$ compact, so that $\kappa$ is strongly compact.

Acknowledgements. Mirna Džamonja thanks the University of Malta for their hospitality during summers of 2017, 2018 and 2019, when this work was done, and the Isaac Newton Institute in Cambridge, UK and Simons Foundation for their support during the Mathematical, Foundational and Computational Aspects of the Higher Infinite (HIF) programme, where she learned about the square compactness from Istvan Juhász. Finally, we thank the anonymous referee for the careful and prompt referee report.

\section{REFERENCES}

[1] B. Cody, M. Gitik, J.D. Hamkins and J.A. Schanker, The least weakly compact cardinal can be unfoldable, weakly measurable and nearly $\theta$-supercompact, Archive for Mathematical Logic, 54, Issue 5-6 (2015), 491510.

[2] A. Hajnal and I. Juhász, On square-compact cardinals, Periodica Mathematica Hungarica 3, Issue 3 - 4 (1973), 285-288.

[3] W. P. Hanf, On a problem of Erdös and Tarski, Fundamenta Mathematicae 53, (1964), 325-334.

[4] P. Holy and P. Schlicht, A hierarchy of Ramsey-like cardinals, Fundamenta Mathematicae 242, Issue 1 (2018), 49-74.

[5] A. Kanamori, The Higher Infinite, Springer Monographs in Math., Springer, 2009.

[6] H. Keisler and A. Tarski, From accessible to inaccessible cardinals (Results holding for all accessible cardinal numbers and the problem of their extension to inaccessible ones), Fundamenta Mathematicae 53, Issue 3 (1964), 225-308. 
[7] P. Lücke, L. Motto Ros and P. Schlicht, The Hurewicz dichotomy for generalized Baire spaces, Israel Journal of Mathematics, 216 (2016), 973-1022.

[8] L. Motto Ros, The descriptive set-theoretical complexity of the embeddability relation on models of large size, Annals of Pure and Applied Logic, 164 (2013), 1454-1492.

[9] J. Mycielski, Two remarks on Tychonoff's product theorem, Bull. Acad. Polan. Sci. Ser. Sci. Math. Astr. Phys. 12 (1964), 439-441.

[10] J.A. Schanker, Partial near supercompactness, Annals of Pure and Applied Logic, 164 (2013), pg. 67-85.

[11] E. Schechter, Handbook of Analysis and Its Foundations, Academic Press, 1996.

[12] P. A. White, Some Intuition Behind Large Cardinal Axioms, Thier Characterization, and Related Results, M.Sc. Dissertation, VCU Virginia, 2019.

David Buhagiar, Department of Mathematics, Faculty of Science, UniVersity of Malta, Msida MSD2080, Malta

E-mail address: david.buhagiar@um.edu.mt

Mirna Džamonja, School of Mathematics, University of East Anglia, NORWICH NR4 7TJ, UK

E-mail address: M.Dzamonja@uea.ac.uk 